\title{
Comparison of SiLi and SDD Detectors for Pharmaceutical Applications
}

\author{
R. A. Carlton \\ GlaxoSmithKline, King of Prussia, PA 19406
}

Silicon Drift Detectors (SDD) have a number of advantages over Lithium Drifted Silicon (SiLi) detectors in energy dispersive spectrometry [1]. The primary advantage is the high count rates of SDD compared with SiLi detectors. The majority of the applications have been to non-beam sensitive, high atomic number materials such as metals, ceramics and minerals. There has been some question as to the possible advantages of SDDs to low atomic number and beam sensitive materials such as pharmaceuticals. The purpose of this investigation is to evaluate SDDs for pharmaceutical applications and to the generation of EDS maps of pharmaceutical tablets.

The following five pharmaceutical tablets were evaluated: a multivitamin, generic naproxen sodium, aspirin, GSK tablet A, and GSK tablet B. All of the samples contained significant amounts of $\mathrm{C}$ and $\mathrm{O}$ based chemicals along with $\mathrm{C}, \mathrm{P}, \mathrm{Si}, \mathrm{Mg}$, and $\mathrm{Cl}$ among others. The tablets were tested using an EDAX Phoenix SiLi detector on an Hitachi 3500N variable pressure SEM. The tablets were sectioned on a microtome and mounted with carbon paste onto an Al SEM stub. The surfaces were examined at $15 \mathrm{kV}$ accelerating voltage in low pressure $(60 \mathrm{~Pa})$. The beam current was determined using a Faraday cage at high vacuum. SDD detectors (with a variety of sizes) from 3 manufacturers were evaluated. In every case, similar beam currents were used.

Figure 1 presents a graph relating x-ray counts per second to beam current for the SiLi (10 $\left.\mathrm{mm}^{2}\right)$ and two SDD detectors (same manufacturer, $10 \mathrm{~mm}^{2}$ and $30 \mathrm{~mm}^{2}$ ) for the sample of naproxen and GSK tablet A. At the same beam currents, both SDDs exhibit clear advantages over the SiLi in count rates. The $10 \mathrm{~mm}^{2} \mathrm{SDD}$ exhibits approximately a 7-fold increase in count rates whereas the $30 \mathrm{~mm}^{2} \mathrm{SDD}$ has approximately a 15 -fold count rate increase. The naproxen tablet contains $\mathrm{C}, \mathrm{O}$, $\mathrm{Na}, \mathrm{Mg}$ and $\mathrm{Si}$. GSK tablet A contains these elements along with $\mathrm{P}, \mathrm{S}$ and $\mathrm{Ca}$.

Figure 2 presents maps of magnesium and a composite elemental map for GSK tablet A using optimum collection conditions with the SDD $30 \mathrm{~mm}^{2}$ detector. This map was collected in less than 3 minutes. Comparable maps using the SiLi detector required more than 20 minutes to collect. The magnesium map is important for assessing the quality of the tablet and this type of map is one of the only ways of determining $\mathrm{Mg}$ distribution.

SDDs have distinct advantages over SiLi detectors in pharmaceutical applications. These advantages include increased count rates, larger detector sizes, and no need for liquid nitrogen.

My thanks to Ted Juzwak of Bruker, Bob Anderhalt of EDAX, and Glenn Kinnear of Oxford for help with this comparison.

\section{REFERENCE}

[1] D. Newbury, et al Microscopy and Microanalysis, 13 (Suppl 2) 2007, pp 1340 - 1 CD. 


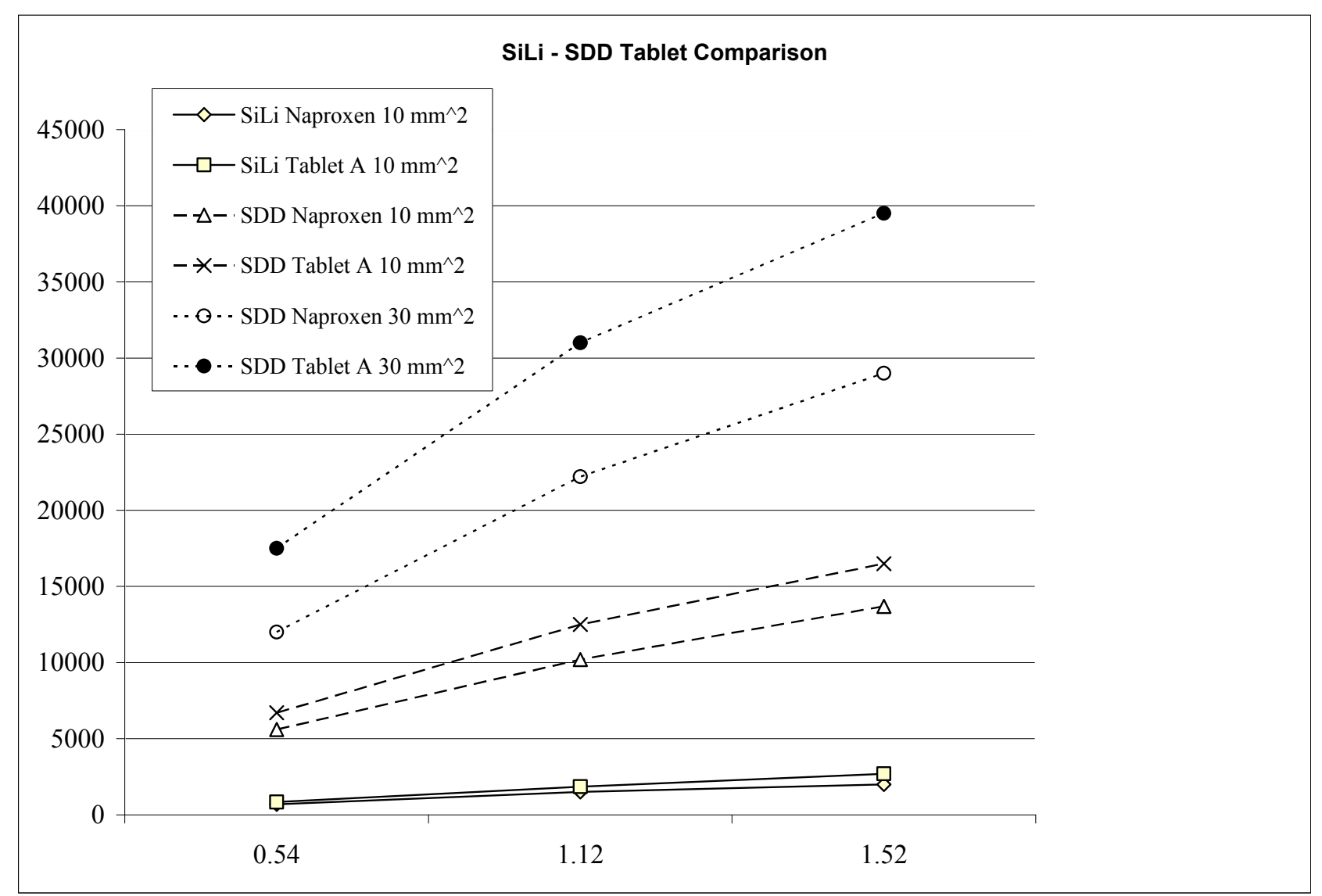

FIG. 1 X-ray count rate versus beam current (nA) detector comparison for naproxen sodium and for GSK Tablet A. More than 7-fold increase in count rate for SDD compared to SiLi (both $10 \mathrm{~mm}^{2}$ ).
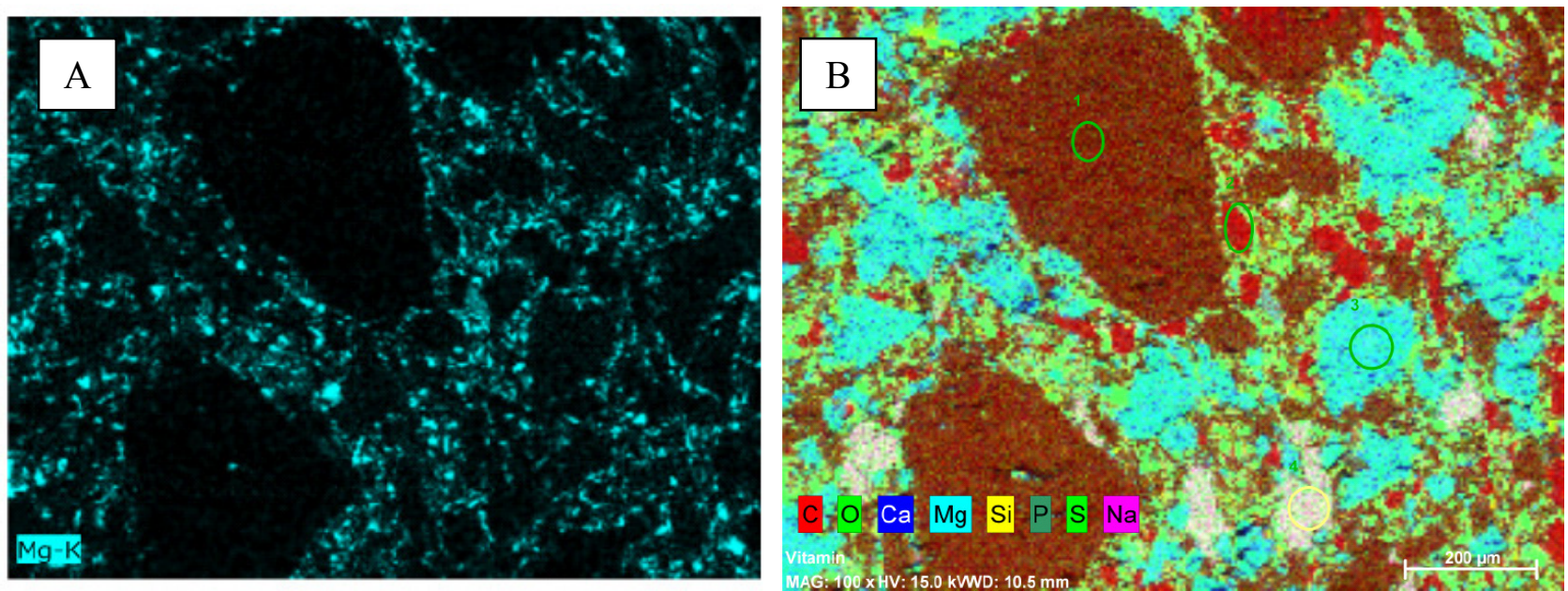

FIG. 2 SDD map of GSK Tablet A. Image A is a magnesium map generated in less than 3 minutes (>20 minutes for comparable SiLi map). Magnesium compound is considered quality critical component and this technique allows for accurate comparisons of $\mathrm{Mg}$ distribution among different tablet lots. Image B is a composite map for all of the elements. 